\title{
K. K. Machanzic
}

NEWFOUNDLA ND July-August 1921.

10000 Botrychium Lunaria (L.) Sw. (Griscom only)

1000 Botrychium virginianum (T.) Swartz

var. Laurentianum Butters Rhodora 19:208.

10002 "Osmunda regalis I. var. pumila Milda" Fernald in

Rhodora 13:132-3. Weatherby calls Am. plant var.

speotabilis (W1Ild.) Gray in Rhodora 21:176.

10003 Osmunda cinnsmomea $L$.

10004 Osmunda Claytoniane I.

10005 Schizaer pusilla Pursh.

10006 Pteretis nodulosa (Michx.) Nieuwl. Rhodora 17:161

(21:175) Am. Midl. Nataral. 3:194

10007 Woodsia glabella k. Br.

10008 pilix bulbifera (I.) onderw.

10009 Polystichum Braunil (spenner) Fet

10010 Thelypetris Filix-mas (I.) Nieuwl.

10011 "spinalosa (Mull.) Euntze var. americana

(Fischer) Weatherby \&f (khodora $\frac{1}{2} 1: 4818$ )

$10012 \pi$ intermedia (Muhl.) Hieuwl. (Port-a-Port)

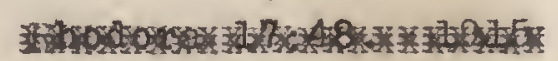

10013 Phegopteris Dryopteris (L.) Fo

$10014 \quad$ Robertiana (Hoffm.) R. Br.

10015 Aspienium viride Hudson

10016 Athyrium thelypteroldes (Michx.) Desv. (First authentic ro

10017 Adiatum pedatum I. var. aleutioum Rupr.

10018 Pteridium aquilinum (L.) Kuhn. See khodora 21:177.

10019 Equisetum arvense s.

10020

10021

10022

10023

10024

Lycopodium Selago ts.

" var. patens (Beauv.) Desv. 


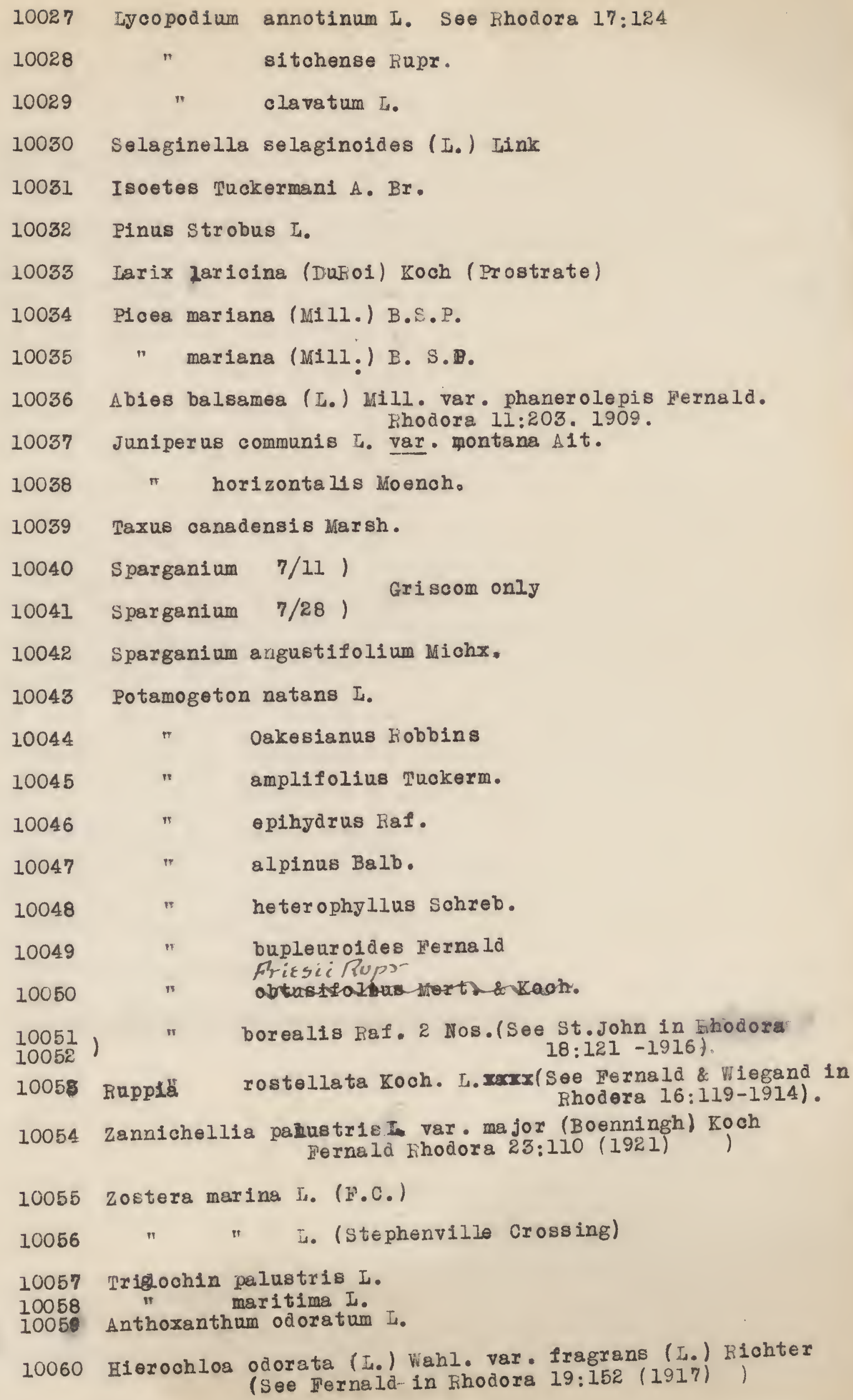




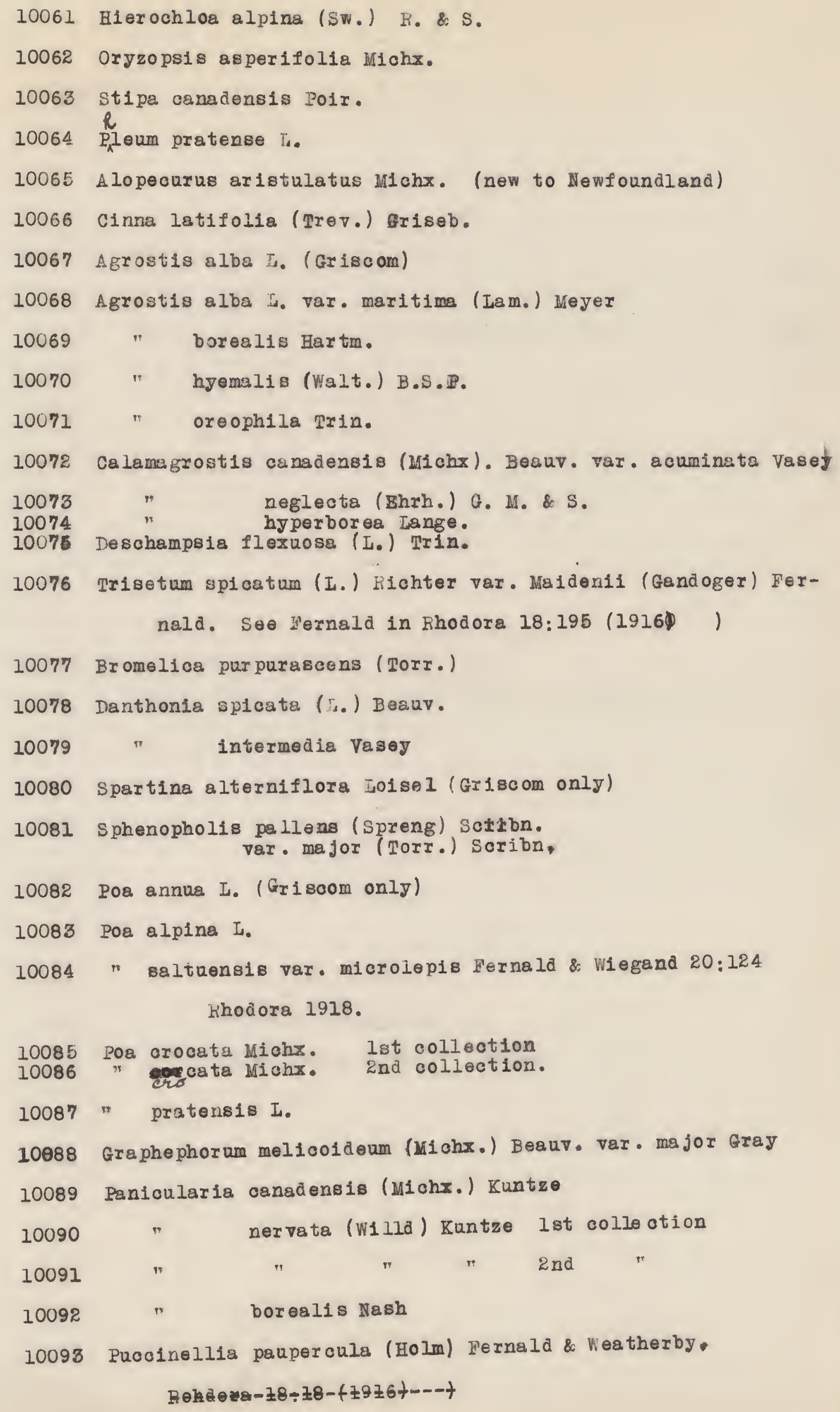




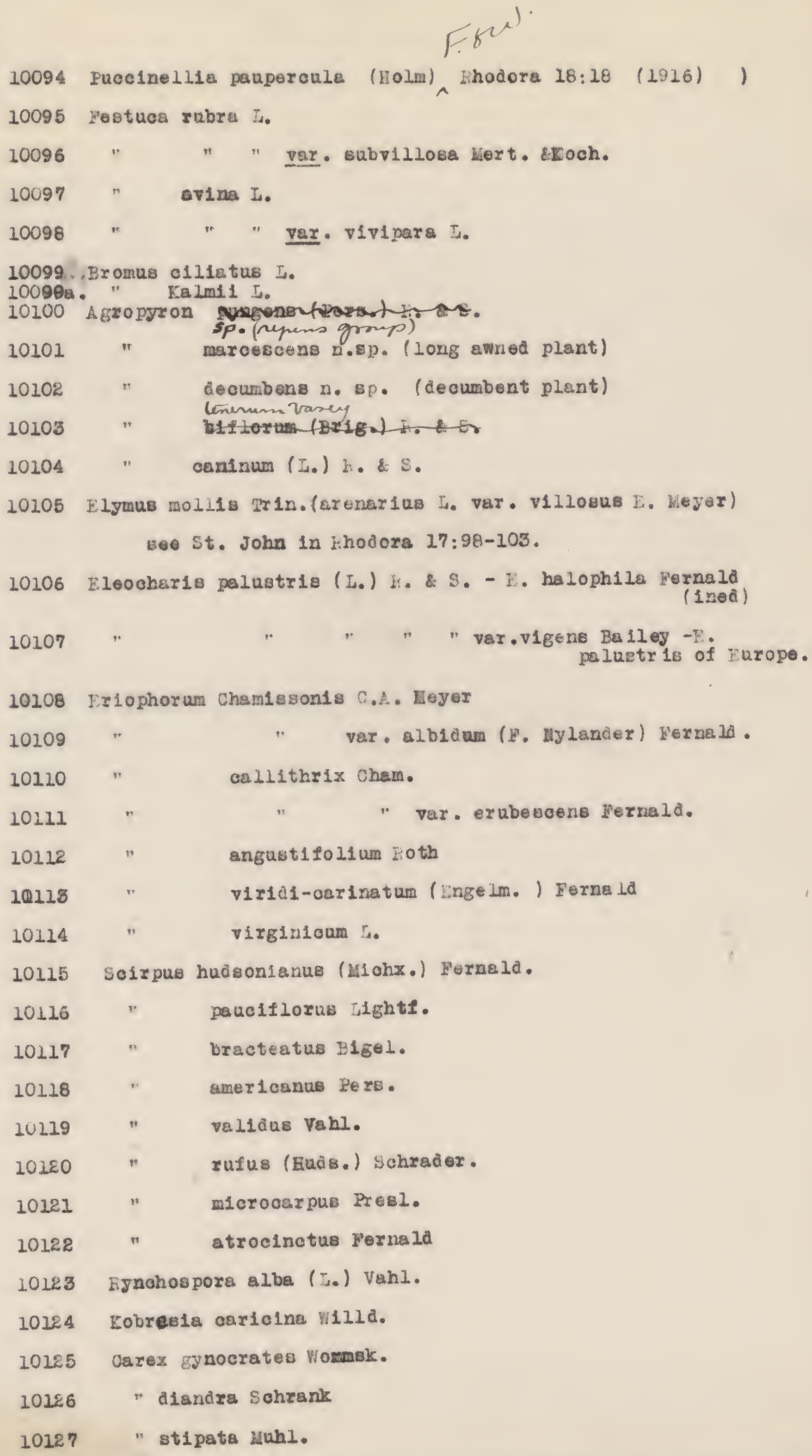




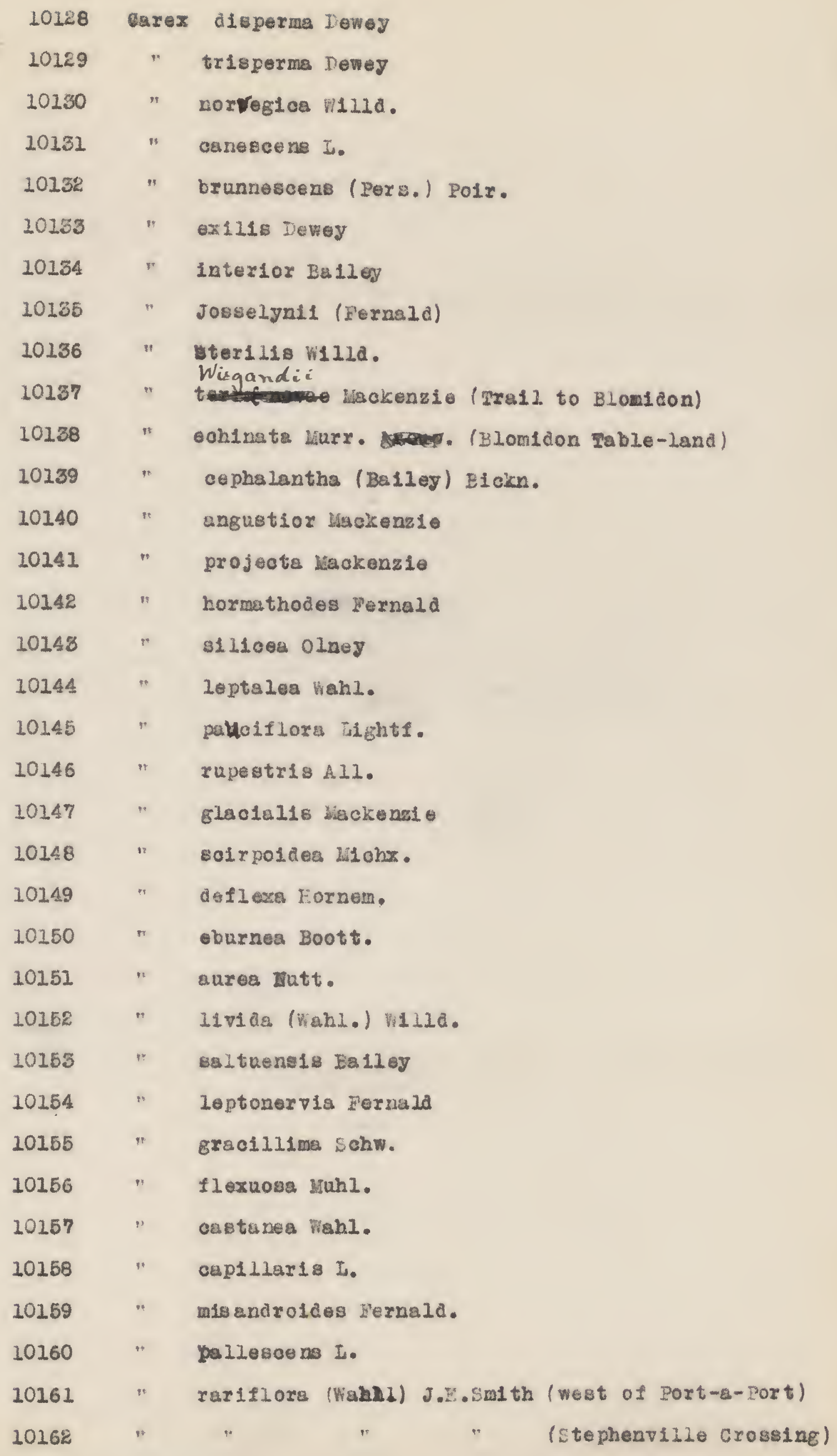




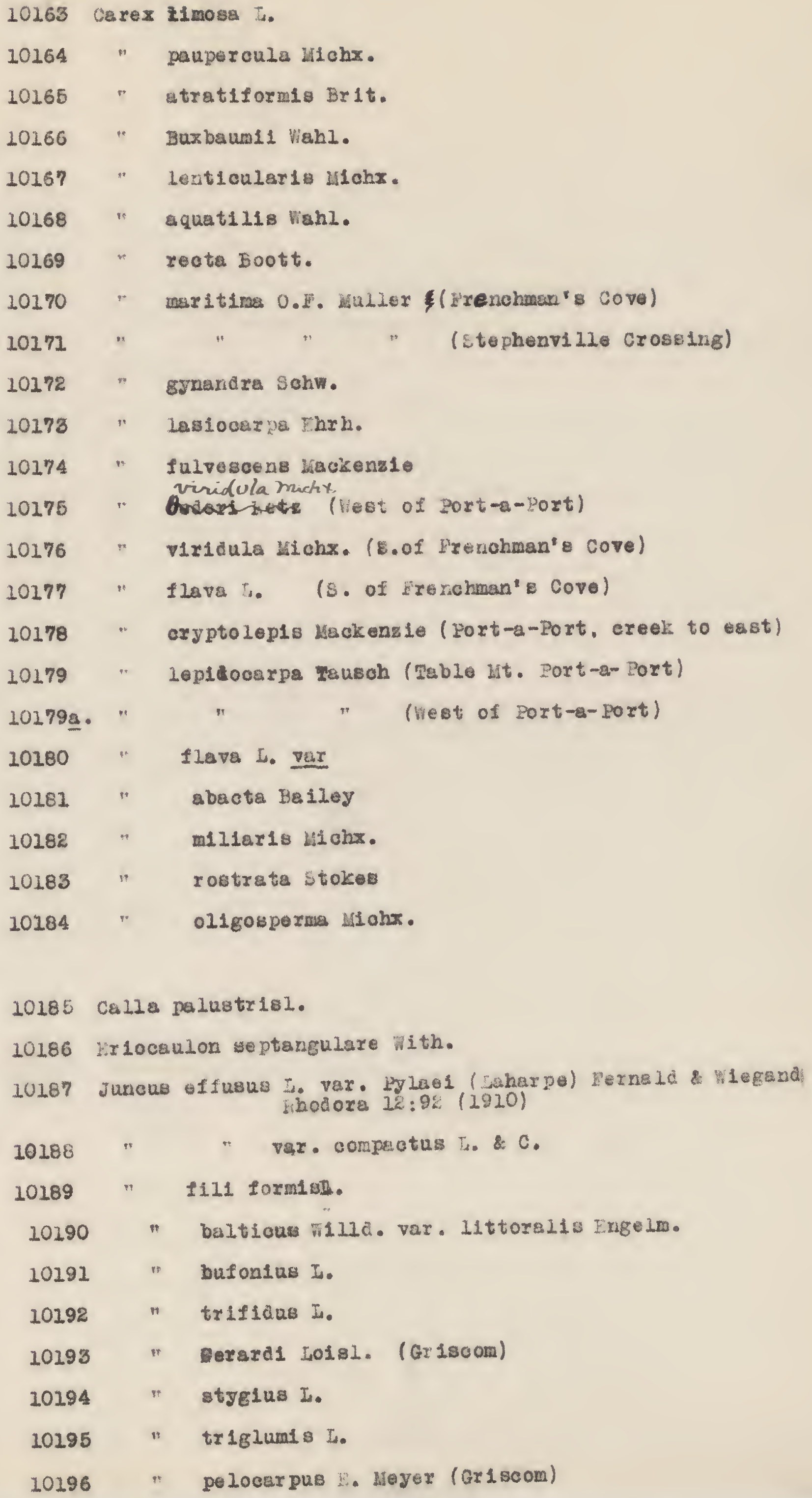




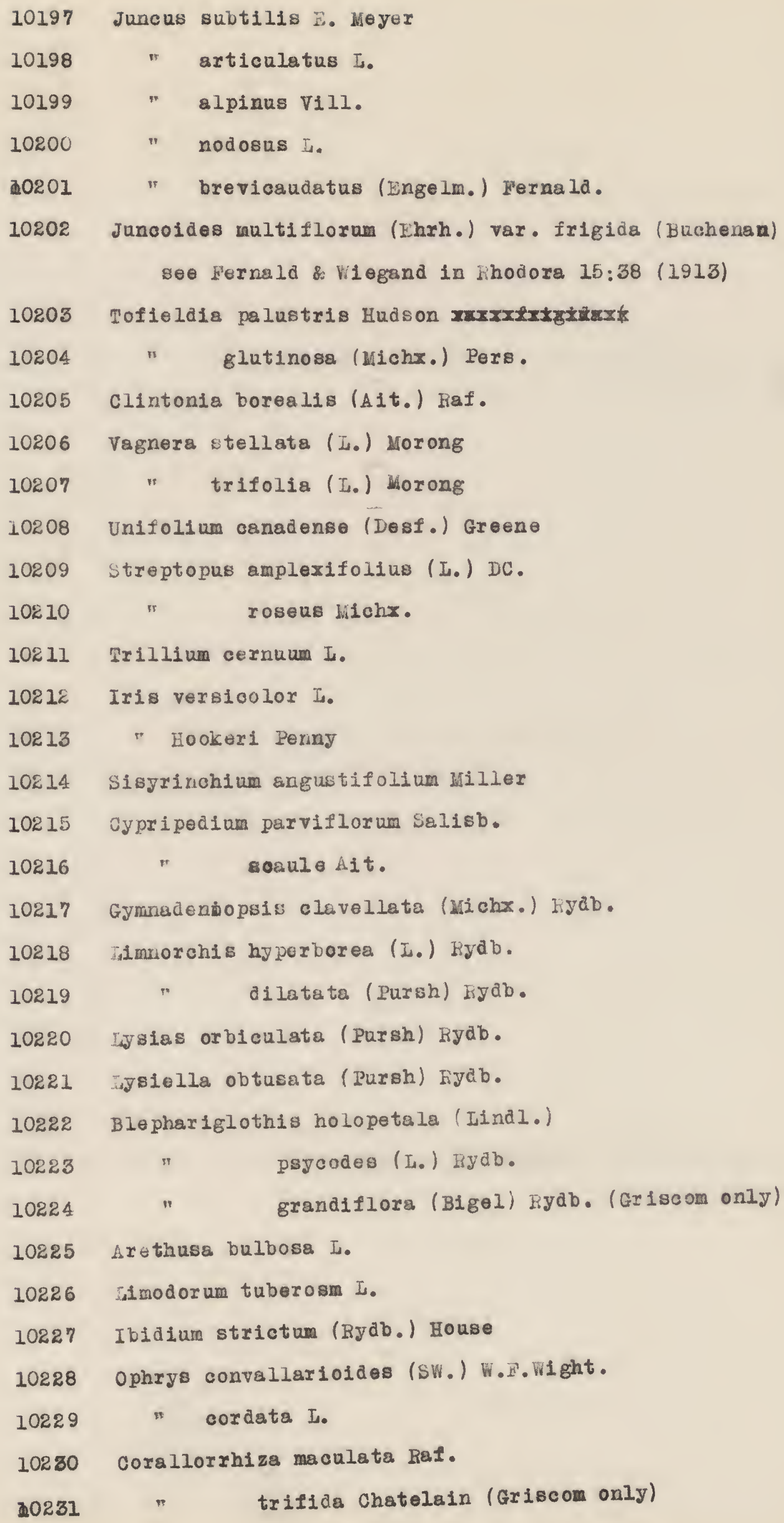




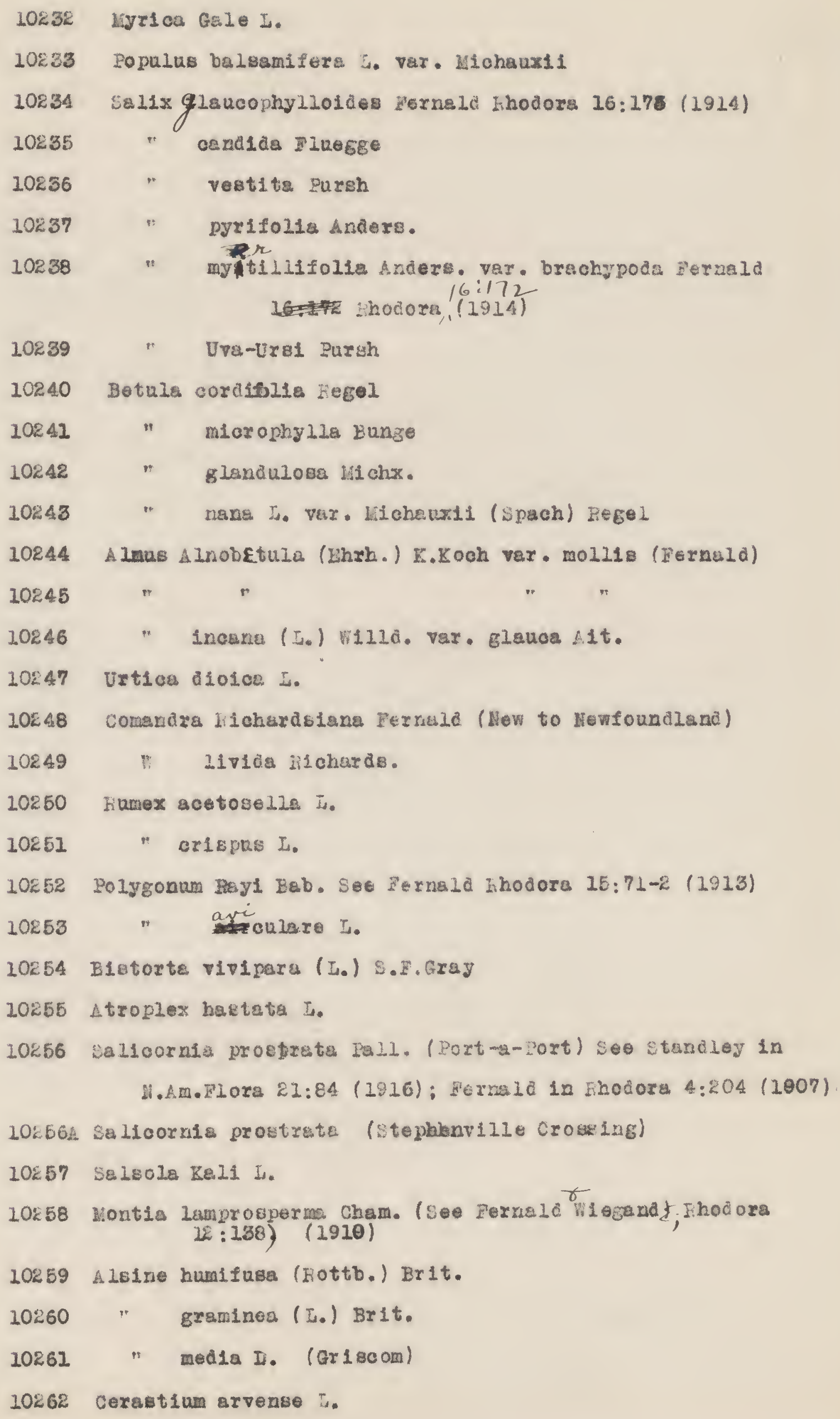


10263

10264

10265

10266

10267

10868

10269

10270

10271

10272

10273

10274

10275

$$
\text { soo Fernald \& St.John, } 1921 .
$$

litoree FGrald (onstted)

$$
\text { Hhodora 11:157\$163 (1910) }
$$

Luchnis alpins $I$. soo Fernald \& st.John! 1921 .
Ihodora 16:43 (1914)

marcescens Ferneld thocora 21:15 (1910)

verras T. var, pubescens (Cham. \& Schlecht) Fernala .

Hochringie latoriflorg ( $\left.H_{0}\right)$ Bersl.

Honkenya maxitima (liaf.) (Soe Fornald in hodora 11:109-115

\$1lone ackul1s is. var exseapa (A11.) no. thodore $23: 1200$

Iymphozanthue varlegatus (Engelm.) Fernald (Fhodore)

$$
\text { 21:187 (1919) }
$$

10276

$$
(\text { Port-a-port) }
$$

10277 coptis trifolla (I.) Salisb.

10278 Actaea rubra (A1t.) willd.

10279 Axemone partiflora Hichx.

10280 lanunculus reptans 5.

10881

ebortivus I.

10282

aorie I.

10283

10284

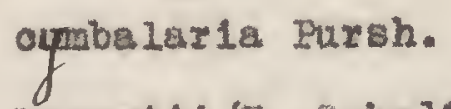

Batrachlum Irouetil(F. Sohulfz) Mym. Seo Exitton lanual

10285 Thaliotrum alpinum is.

10286

10287

polygatsus turi.

10288 Iraba arabians Michx.

( Dhordona $7: 65$ )

10889 " arsbisans Michx . vex . orthocarpe Fernsla \& Knowlton

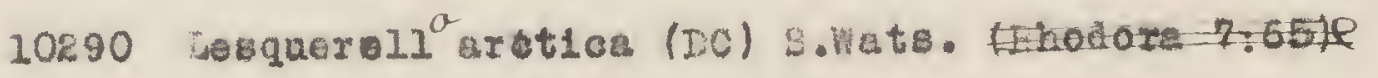

10291 Buxsa Bursa-nsstorí (I.) Brit.

10292 Thlagpi arvense

10293 Cochlearle officlnelio I.

20294 Carảamino pennsylvanica Luhl. 


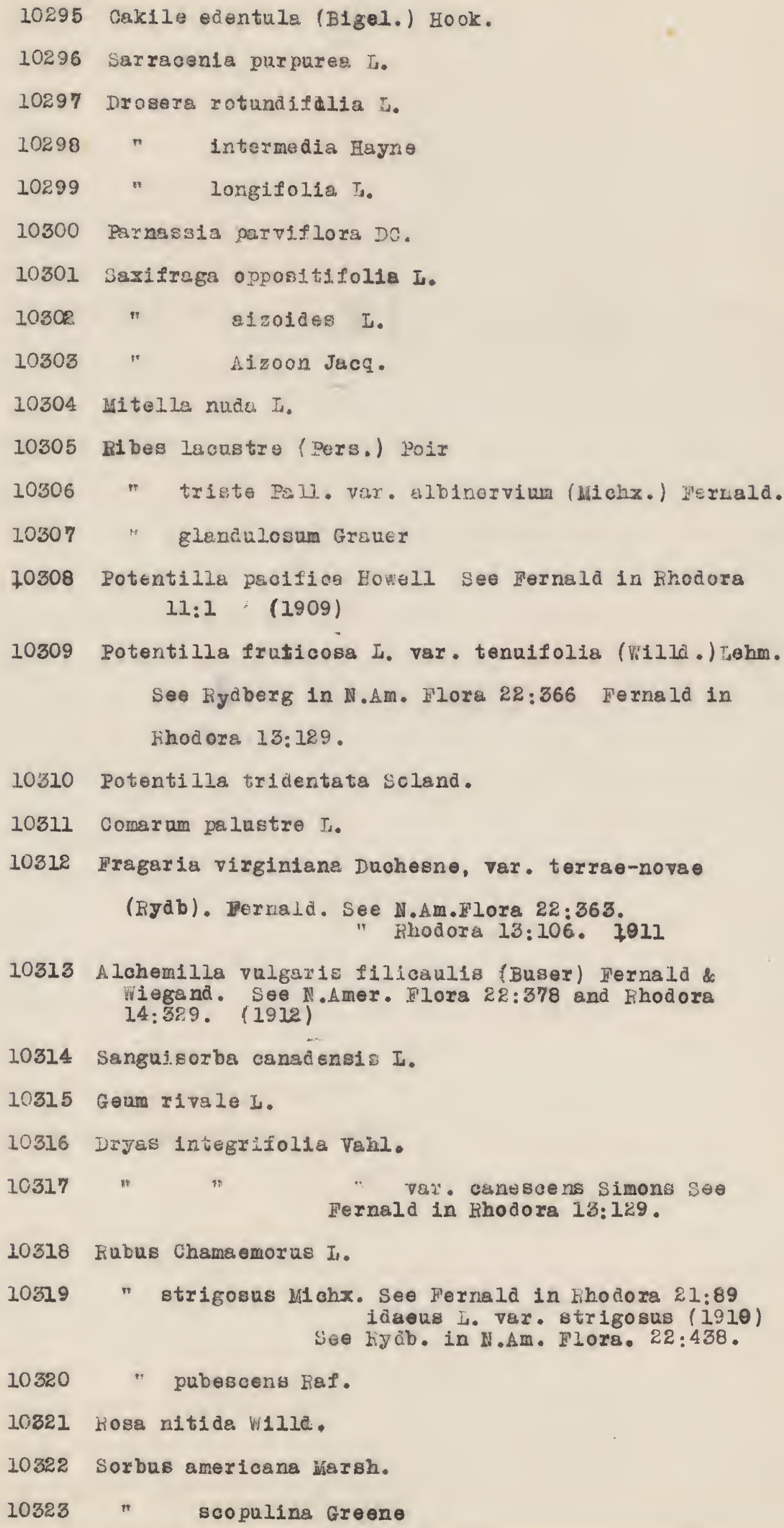




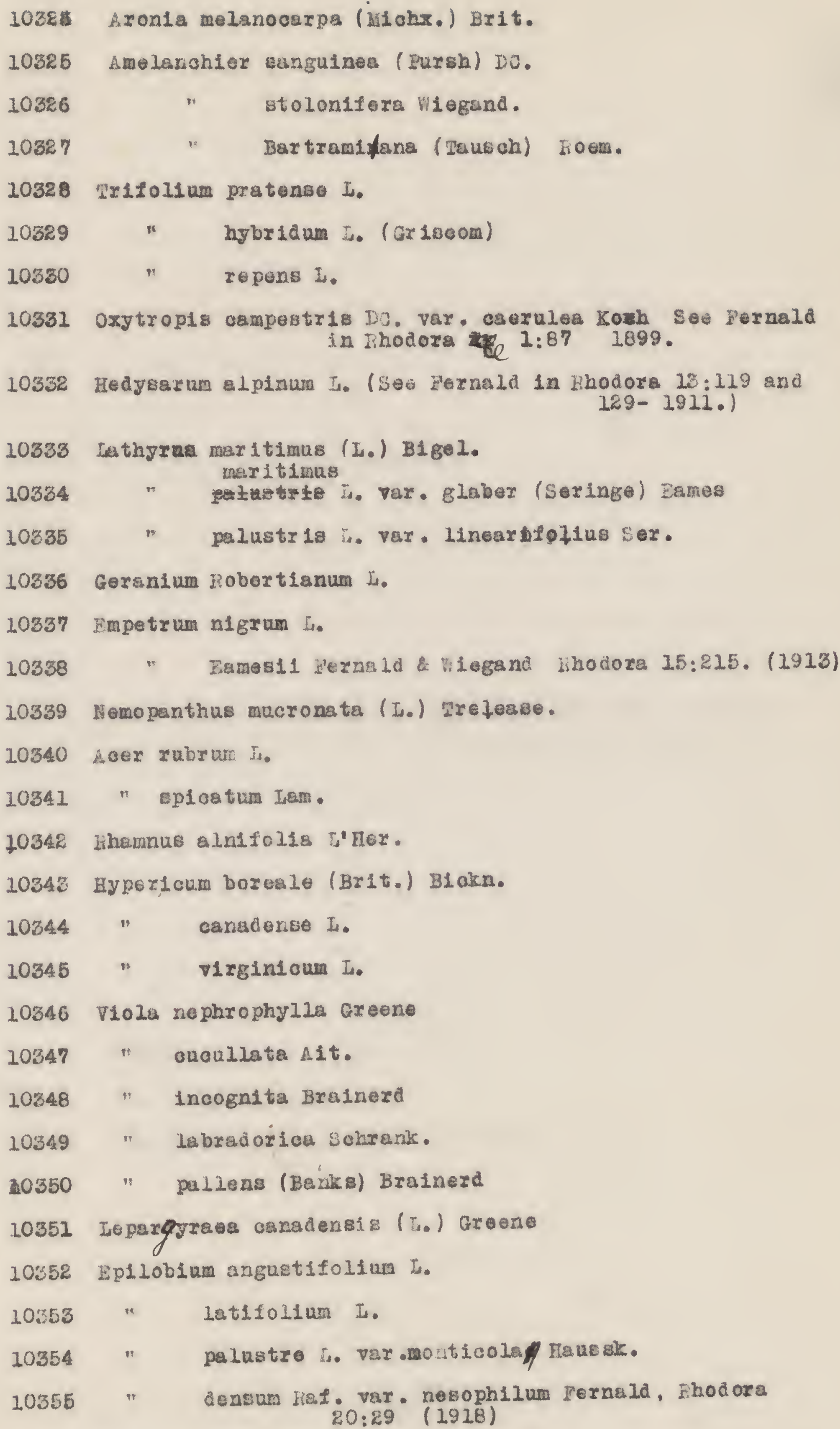




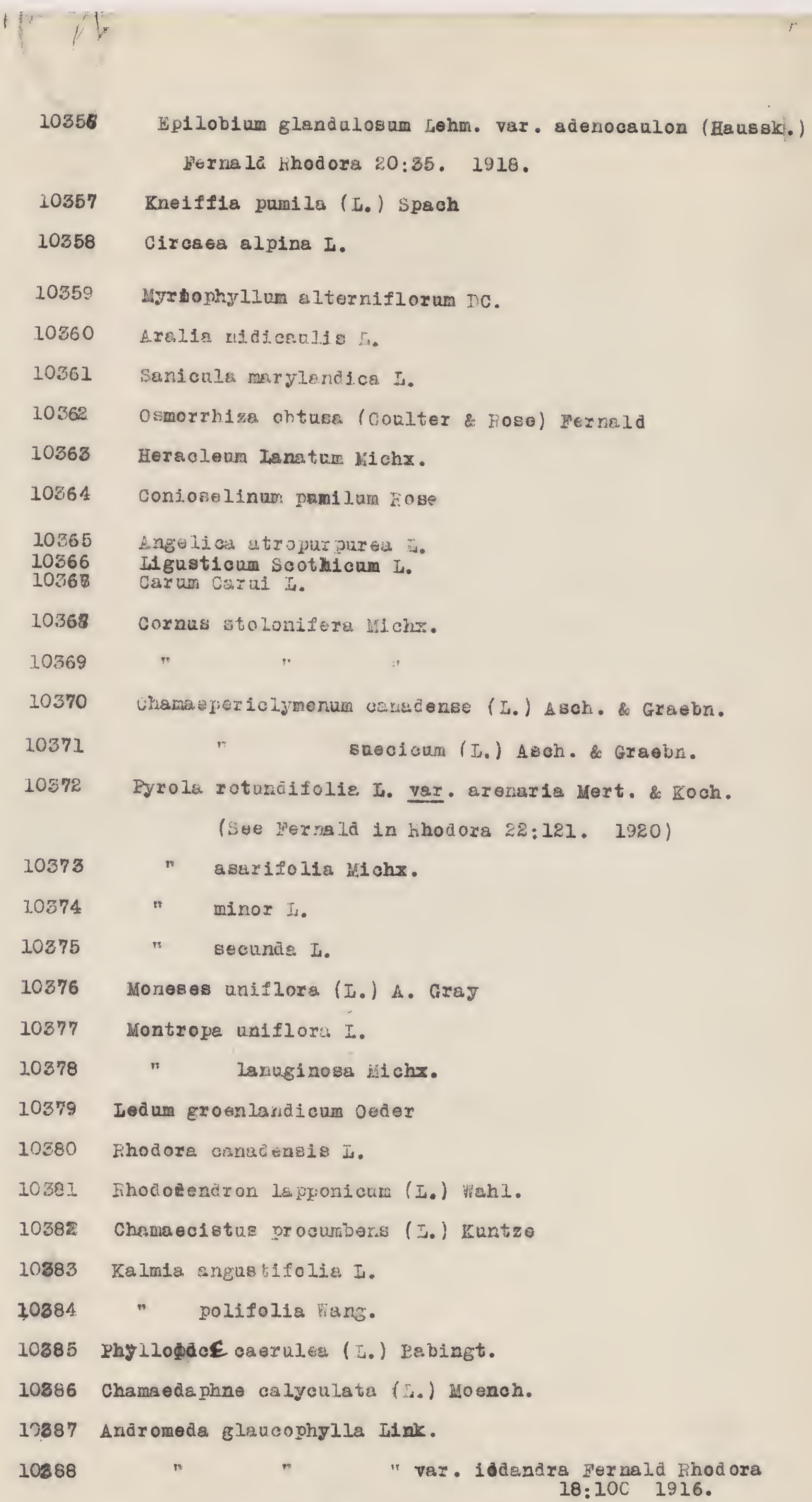




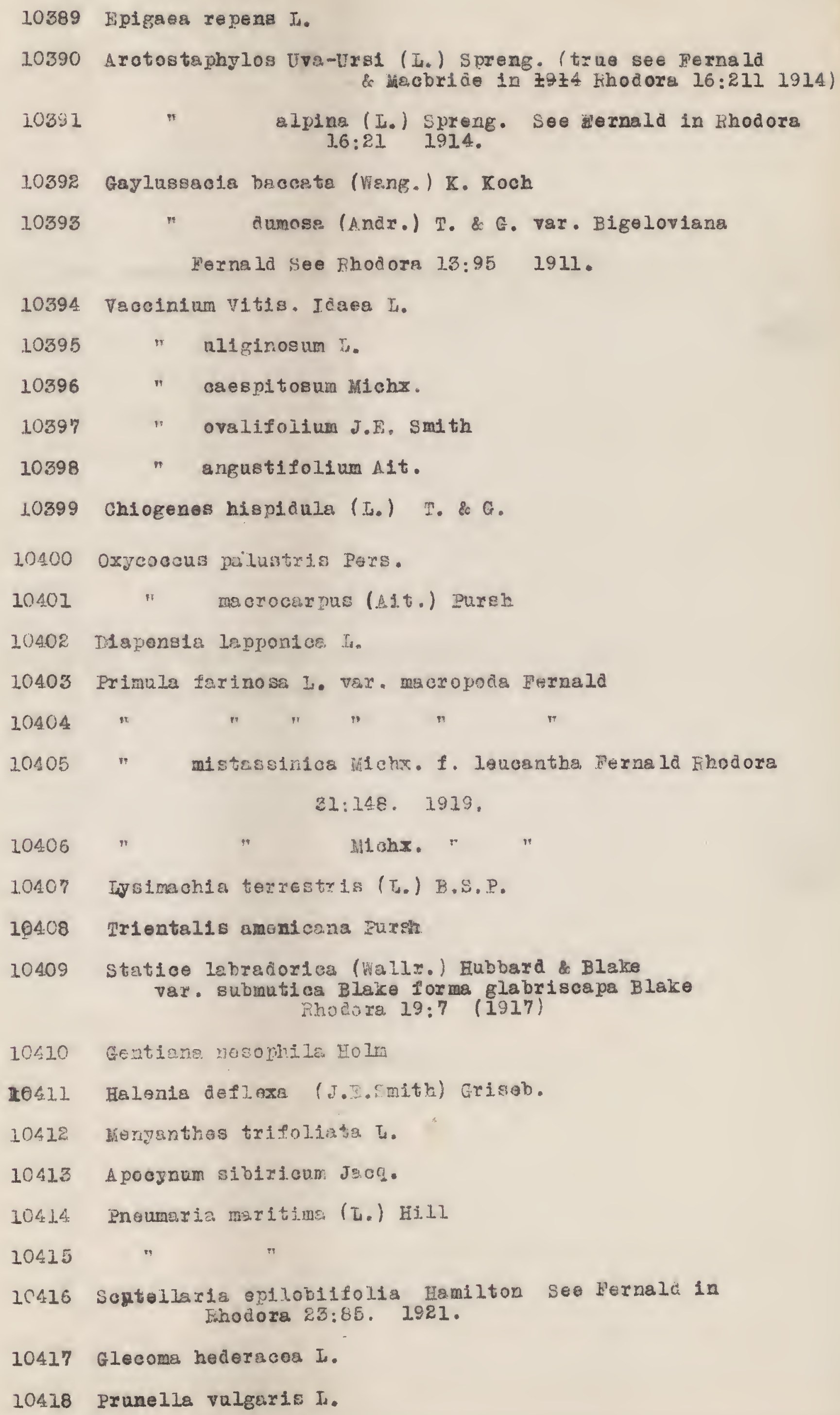


10419

10420

10421 Veroniea amertcsue schw.

104 Castilleja scuminste (Eursh) spreng.

10423 vurixasia amerioana vettst. soe Fernala \& Wiggand

10424 purpurea kooks

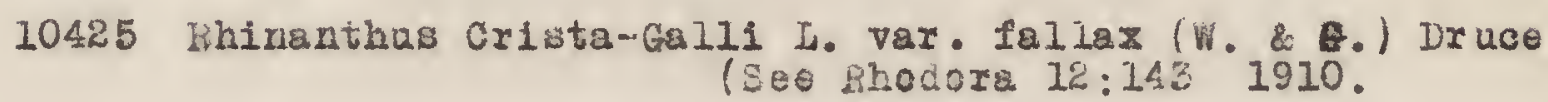

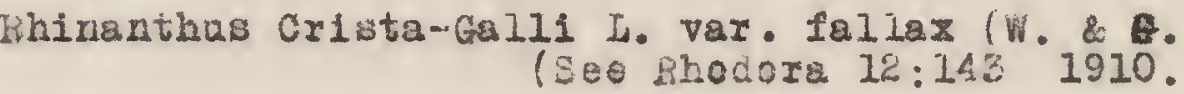

10426

10427

10428

1042

10430

10431

10432

10433

10434

10435

10436

10437

10438

$10 \% 39$

204.40

10441

10442

$10 t_{x} 43$

10444

1044.5

10446

1044.7

10448

10449

10450

10451

Fhodora 17:181 1915. ot longifollus seragld

Lelampyrum Inseare Lam.

Finguioula vulgario L.

vtrionlaria Intermodia Hayno

$"$ minorI.

macroritive jevorte

" sorriata ziohx.

Thalesia unifzora (I.) Brit.

Plantago major : (Griocom)

Plantago maritimals.

Galium Kamtschaticun Steller

" triflorum sickx.

" lakradortent Wieg.

"Claytonl vitoht. var. subbiflorum wiegenả

$$
\text { Rhodora } 12: 229 \text { (1910) }
$$

n palustre I.

r acprellar Micho

Sลmbu

Viburnum penofflorum Pylaio

" osesinotoses.

Linn:ea amexicens forbes

icena amorulan . var. calvescens Feruald \& wigand Khodora 12:210 (1910)

Valerisna ska septertrionalis Rydb.

Comprnula rotundifolia L.

Lobelia Dostuana I. 
1045

10453 Apargia autumnale (L.) Hoffm. var. pratensis (Link.)

10454 Hieraciun canadense Michx.

10455 Nabelus nanad BiфgeI) porreg

10456 Eapetorium Bruner 1 A. Gray

10457 soliago hispida wuhl. For varioties see fernald

$$
\text { Lihodora 1\%:1-3 (1915) }
$$

10458 macrophylla Pursh var. thyrsoldea (Mey.) fernald

10459 multiracista Ait.

10460 " uliginose Nutt.

10461 Aster follaceas rindl. For ver. see Fernald Rhodora 17:15

10462 "radula Ait.

10463 "nemoralis Ait.

10464 Erigeron hyssopifolius Mlchx.

10465

10466 Antennaria eucosma ferrsld \& Wiegand (Khodora 13:23. 1911)

$10467 \quad$ eana (Fernald \& Wiegand)(Khodora $12: 241911$ )

10468 spathulata Fernala (Fhodora 16:132 1914)

10469 Anap/ihalis margaritacea (I.) B. \& H. See Ferrald in var. occidertalis Greene 1911.

10470

10471

10472

10473

10474

10475

10476

10477 Arnica chlonopappa Fernald

10478 Sonecio vulgaris $\mathrm{L}$.

10479 " pseudo-Arniog toss.

10480 "aurens $\mathrm{I}$.

10481 "paperoulus Michx. (Blomiäon)

10482

10483

1915.

Rudbeckia birta I. var. occidentalis Greane

Achillon millefolium t.

" subalpina Greene (kydberg in N.An.Flora 34:222.

" " $"$ forms rosea

Chrysanthemum Ieucanthemum t. var. pinnatifiaum Iecog

Artemisia candata Michx. or Canademsors

borealis Pall. var. Purshii Besser (Seè Pyabex in N.Am. ilora 34:255. 1916). 
10484 Senocio Fernaldil forma lingulatus Fornald

$$
\text { Rhocore 18:155. (1917) }
$$

10485 Cirsium muticum Michx.

10486

10487

Nova Scotia Senecio Jacobaes I. 